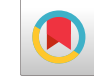

\title{
Investigating Differential Expression of mTOR1/UCA1 in Tumor Samples of Colorectal Cancer Compared with Tumor Marginal Samples
}

\author{
Maryam Alamdar ${ }^{1,}{ }^{*}$ and Majid Sadeghizadeh ${ }^{2}$ \\ ${ }^{1}$ Department of Genetics Sciences, Factually of Advanced Sciences and Technology, Tehran Medical Sciences, Islamic Azad University, Tehran, Iran \\ ${ }^{2}$ Department of Genetics, Factually of Biological Sciences, Tarbiat Modares University, Tehran, Iran \\ "Corresponding author: Department of Genetics Sciences, Factually of Advanced Sciences and Technology, Tehran Medical Sciences, Islamic Azad University, Tehran, Iran. Tel: \\ +98-9196303025, Fax: +98-2122006660, Email: maryam.alamdar95@gmail.com
}

Received 2021 July 25; Revised 2021 October 12; Accepted 2021 October 31.

\begin{abstract}
Background: Colorectal cancer (CRC) is the second and third most common cancer in men and women respectively, and the fourth cause of cancer death of individuals. Mutations in specific genes can lead to colorectal cancer. UCA1 is one of the oncogenic genes that have been shown to stimulate cell proliferation. mTOR1 is another gene that leads to the growth of cancer cells through anabolic processes and autophagy inhibition.

Objectives: In this study, we evaluate the expression of these two genes in different phases of CRC, that helps the early detection of colorectal cancer which can increase the survival rate.

Methods: First, we collected 25 colorectal cancer tumor tissues and 25 adjacent normal tissues as a control group. Then, RNA was extracted from tissue samples and cDNA synthesized. The UCA1 and mTOR1 expression was evaluated in CRC tissues compared to adjacent normal tissues by Real Time PCR.

Results: Our results showed that the UCA1 and mTOR1 expression in the tumor tissues was significantly higher than in the adjacent normal tissues $(\mathrm{P}<0.05)$. There was also a significant difference in Lynph inv and Vescu inv with mTOR1 expression $(\mathrm{P}<0.05)$.

Conclusions: Our results showed that UCA1/mTOR1 may be important genes involved in colorectal cancer. mTOR1 was also identified as one of the possible genes in metastasis of colorectal cancer. Thus, UCA1 and mTOR1 can probably be considered as biomarkers in CRC therapy and diagnosis.
\end{abstract}

Keywords: Colorectal Cancer, mTOR/ UCA1, Biomarker

\section{Background}

Colorectal cancer (CRC) is the second and third most common cancer in males and females, respectively (1). The incidence of colorectal cancer has increased from 1991 to 2012 by more than 200,000 new cases per year. Most people with colorectal cancer are found in Western countries (55\%); however this is changing due to the rapid development of some countries over the past few years (2). CRC is a multifactorial disease in which genetic and environmental factors together play an essential role as risk factors. Age, gender, ethnicity, heredity and family history, physical activity, overweight and obesity, diet, tobacco, and alcohol consumption are important environmental risk factors (3). Studies reveal that most people are at the highest risk for colorectal cancer at age 50 and the colorectal cancer risk decreases after age 50 (4). A study of both men and women has demonstrated that males are at greater risk to CRC for high-risk lifestyle (5). Among the various races, blacks have the highest amount of colorectal cancer (6-8). High physical activity greatly reduces the risks of colorectal cancer. Moreover, physical activity has directly related to reducing obesity and overweight (9). Tobacco and alcohol have recognized among the most important CRC risk factors for toxic and carcinogenic substances (10). However, hereditary factors also expose the persons to CRC. The familial syndromes, and a family history of polyps and intestinal adenomas in first- or second-degree family members increases the risk of CRC (11).

Diagnosis of the CRC in the early stages strongly has affected the appropriate treatment of colorectal cancer. The various screening tests, such as biomarkers, gene expression profiles, computed tomographic colonography (CTC), and other modern methods, have developed to help early 
diagnosis and reduce mortality of CRC (3).

CRC has misled symptoms such as abdominal pain, mood swings, and anemia, which may be seen in other inflammatory diseases $(12,13)$. Common colorectal cancer treatments include surgery, chemotherapy, and radiotherapy, which are used according to the CRC stage (14). So, screening tests are essential for CRC diagnosis and treatment.

Although environmental risk factors are relatively predictive of colorectal cancer, genetic factors and mutations also play an important role as a risk factor. Numerous mutations may be involved in colorectal cancer, the most important of which occurs in the DNA repair system, often involving APC, TP53, and KRAS (15). Among genetic factors, one of the most important mutations occurs in long noncoding RNA (LncRNA). UCA1 mutation disrupts the regulation of the cell cycle. This long non-coding RNA is involved in many oncogenic pathways, is known to be an important factor in drug resistance (16). In cancer cells, UCA1 is stimulated by hypoxia-1 induction factor during oxygen deficiency that under this condition, the secretion of UCA1-rich exosomes increases $(17,18)$. The UCA1 gene also stimulates the cell cycle, and its expression inhibition stops the G0/G1 phase in cancer cells (16). Initially, UCA1 was suggested as a marker for the prognosis and survival of patients with colorectal cancer $(16,19)$. Interestingly, a recent study of tumor-derived exosomes showed that UCA1 expressed not only in gallbladder cancer exosomes (17), but also in exosomes isolated from serum of colorectal cancer patients (20).

Another gene which is involved in colorectal cancer is mTOR1. mTOR1 is an important regulator of cell growth control that is abnormally activated in more than $70 \%$ of cancers (21). mTOR1 acts as a kinase serine/threonine protein and is classically essential for cell growth by regulating protein translation. The mutation in this gene is directly related to the activation of oncogenic pathways (22). mTOR1 is also associated with metabolic development, vascular production, and metastasis. Based on the wide range of cancer features promoted by mTOR1, it is not surprising that cancer cells choose the mTOR1 pathway as a mechanism for their development (23).

Wu et al. showed that LncRNA UCA1 was involved in tamoxifen resistance by activating the mTOR1 pathway, indicating a link between the mTOR1 pathway and LncRNA UCA1 (24). In a study on the role of UCA1 in lung cancer, Wang and Fa, also showed that degradation of UCA1 leads to inhibit viability and glycolysis in NSCLC cells by suppressing PKM2 expression (25).

\section{Objectives}

Considering the relationship between these UCA1 and mTOR1 in different cancers, we evaluated this relationship in Iranian patients with colorectal cancer.

\section{Methods}

\subsection{Tumor Tissues}

25 tumor tissues and adjacent normal tissues from colorectal cancer patients were collected. The average age of patients was 41.3 years (Table 1 ). Written informed consents were obtained from patients of all subjects, and the research protocol was approved by the Ethics Committee of Islamic Azad Tehran Medical Sciences University- Pharmacy and Pharmaceutical Branches Faculty (Approval ID: IR.IAU.PS.REC.1399.085).

\begin{tabular}{|cc}
\hline Table 1. Clinical Information of Patients & \\
\hline Variables & Values \\
\hline Average age & 41.3 \\
\hline Stage of the disease & \\
\hline I & 6 \\
\hline II & 11 \\
\hline IV & 5 \\
\hline Lymphatic invasion & 15 \\
\hline Vascular invasion & 10 \\
\hline Total & 47 \\
\hline
\end{tabular}

\subsection{RNA Extraction and cDNA Synthesis}

According to the company's protocol, total RNA was extracted from tissues using the South Korean company Genall's Trizol solution. The quantity and quality of extracted RNAs were measured using spectrophotometer (A260/280 ratio) and agarose gel electrophoresis. cDNA synthesis was then performed using Takara's cDNA synthesis kit according to the manufacturer's instructions.

\subsection{Real Time PCR}

In order to evaluate the expression of UCA1 and mTOR1, Real Time PCR was performed using primers designed for target genes (Table 2).

\subsection{Statistical Analysis}

The data from the real time PCR reaction were evaluated using Excel and SPSS software. ANOVA and $t$-test were used to analyze the data. In all cases, p-value less than 0.05 was considered as a significant difference. 


\begin{tabular}{lc}
\hline \begin{tabular}{l} 
Table 2. Sequence of Specific Primers for UCA1 and mTOR1 Genes \\
\hline Gene Primers
\end{tabular} & Sequence \\
\hline mTOR1 & \\
Forward & 5'-GGGACAGCATGGAAGAATACA-3' \\
\hline Reverse & 5'-TGTTGTGCCAAGGAGAAGAG-3' \\
\hline UCA1 & \\
\hline Forward & 5'-CCTATCTCCCTTCACTGACTCT-3' \\
\hline Reverse & 5'-GTCCGTATAGAAGACACCCAATC-3' \\
\hline GAPDH & \\
\hline Forward & 5'-CCGAGCCACATCGCACAG-3' \\
Reverse & 5'-GGCAACAATATCCACTTTACCAG-3' \\
\hline
\end{tabular}

\section{Results}

\subsection{UCA1 and mTOR1 are Upregulated in CRC Tissues}

The expression of UCA1 and mTOR1 in tumor tissues and adjacent normal tissues was compared. The analysis showed that UCA1 and mTOR1 genes had high expression levels in tumor tissues in comparison with adjacent normal tissues $(\mathrm{P}<0.05)$ (Figure 1$)$.

First, we evaluated the relationship between the samples in tumor tissues and adjacent normal tissues, and then evaluated the relationship between different groups of each sample separately, for metastasis detection and early detection (Figure 2).

\subsection{UCA1 / mTOR1 Expression in Different Grades of the Tumor}

The expression of UCA1 gene in Grade I and IV, and in Vescu inv and Lynph inv was insignificant $(\mathrm{P}>0.05)$. However, Grade II showed significant changes $(\mathrm{P}<0.05)$.

The mTOR1 at different grades of tumor specimens (grade I, II, IV) as well as in the lymphatic invasion and vascular invasion (Vescu inv and Lynph inv) showed a significant increase in expression $(\mathrm{P}<0.05)$.

\section{Discussion}

In this study, our finding showed that UCA1 expression in tumor tissue was higher than adjacent normal tissues. We also performed the UCA1 expression in different tumor grades, Lynph inv, and Vescu inv. We found that UCA1 expression increased in grade II of colorectal cancer but had no significant changes in Lynph inv, and Vescu inv. These results suggest that UCA1 probably plays a vital role in the development of colorectal cancer. Therefore, UCA1 can probably be considered as a biomarker for the early detection of colorectal cancer.

We also found that mTOR1 expression increased in tumor tissue compared to adjacent normal tissues. Lynph inv and Vescu inv results were significant and mTOR1had higher expression in IV grade of CRC tissues. Therefore, mTOR1 may be correlated with metastasis and cell proliferation in CRC. Therefore, mTOR1 can possibly be used for the early detection of colorectal cancer and metastasis.

LncRNAs play a crucial role in regulating gene expression; therefore they contribute to cancer by affecting cellular homeostasis, including proliferation, survival, migration, and gene stability (26). Recently, LncRNAs have identified as new markers for cancer detection, but operate with different diagnostic accuracy. LncRNAs have suggested as a promising indicator for the diagnosis of colorectal cancer. Mechanisms of how LncRNAs affect CRC development and metastasis are unclear. UCA1 facilitates cell proliferation, and cell cycle progression, and prevents apoptosis in colorectal cancer and is directly related to tumor size (16). Yang et al. Showed that the Inhibition of UCA1 sensitized colorectal cancer to radiotherapy, has increased radiationinduced cell death, and cessation of the cell cycle in the G2 / M phase by inhibiting colony formation proliferation (27). Cui et al. reported that Inhibiting UCA1 and increasing the expression of miR-28-5p together could stop the cell growth and migration. The HOXB3 gene was identified as a regulatory pathway for UCA1 activity that mediated between UCA1 and microRNA. Therefore, UCA1 has suggested as a potential therapeutic target for colorectal cancer (28).

mTOR1 is located downstream of the PI3K/AKT pathway and activates important signaling pathways such as PI3K, MAPK, and AMPK to control cell growth, proliferation, and survival. Given the main role of mTOR1 in cell growth and metabolism, the relationship between mTOR1 pathway activity and cancer can be predicted (29). In each of the mTOR1 signaling pathways, different types of cancers have reported, and generally, mTOR1 has a significant effect on tumor progression (30). Therefore, mTOR1 is an attractive therapeutic goal. Previous studies reported that mTOR1 was more associated with left-sided tumor localization. High frequency and abnormal mTOR1 expression provide the main reason for targeting this pathway, and to treat colorectal cancer patients. However, the role of mTOR1 in the prognosis of colorectal cancer cannot be confirmed and further studies need (31). In a study by Zhang et al., mTOR1 signaling components such as mTOR1, p70s6 K, and 4EBP1 were activated in colorectal cancer cells, and malignant large intestinal adenomas in colorectal cancer. Inhibition of mTOR1 expression using specific siRNAs significantly reduced cell growth in the laboratory and body. The mTOR1 signaling was associated with clinical parameters of colorectal cancer. They suggested silencing the mTOR1 gene through siRNA might be a novel treatment strategy for CRC (32). Gulhati et al. showed that mTOR1 and mTOR2 increased at stage four and distant metastases in colorectal 
mTOR

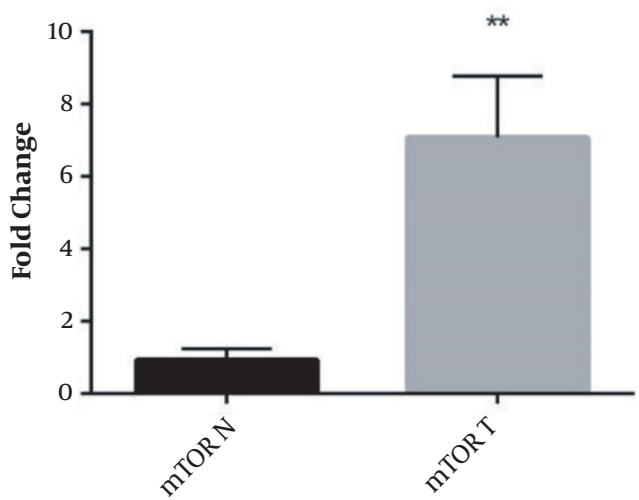

UCA1

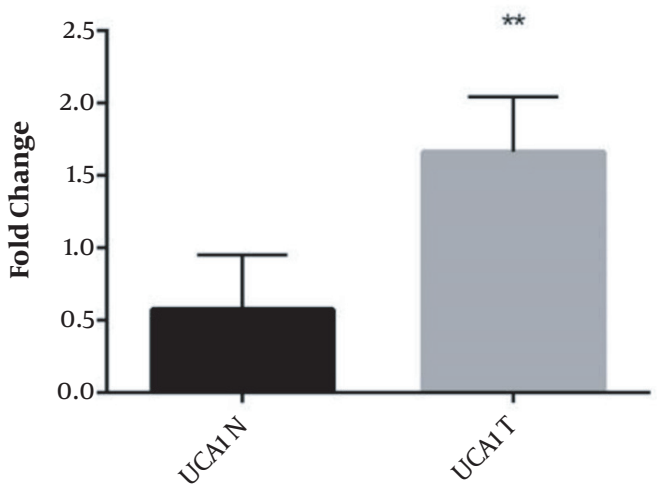

mTOR Network Exprision

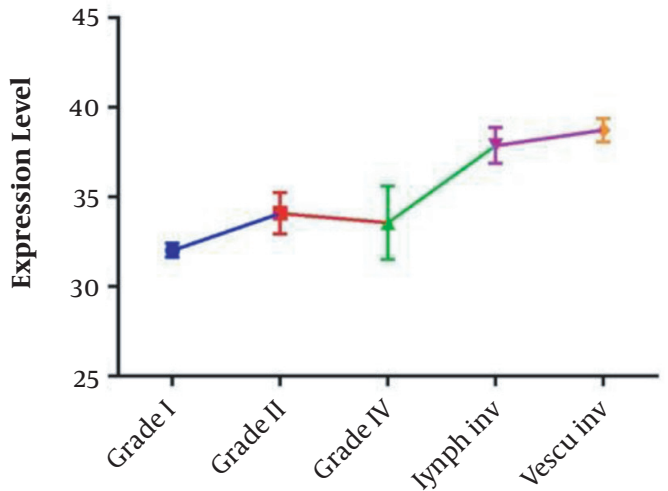

UCA1 Network Exprision

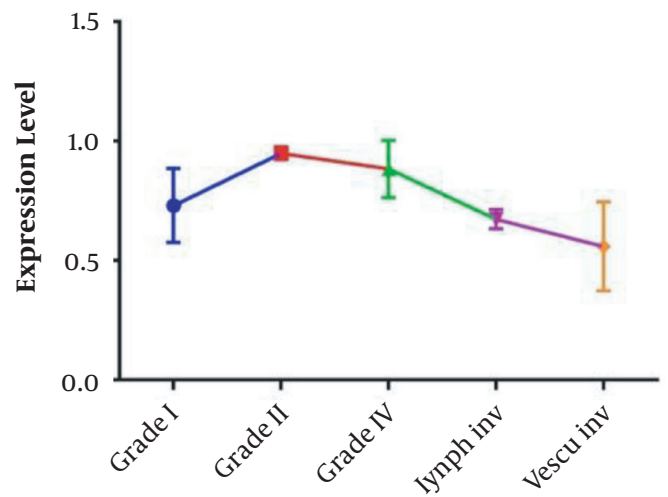

Figure 2. UCA1 and mTOR1 expression in different tumor grades, lynph and vescu inv

cancer. Their findings showed that mTORC1 and mTORC2 were involved in the regulation of epithelial mesenchymal transmission, motility, and metastasis of colorectal cancer through RhoA and Rac1 signaling, and suggested that the mTOR1 and mTOR2 inhibitors could be used as a therapeutic target (33).

The mTOR1 and UCA1 play a vital role in the progression of colorectal cancer and may be used as appropriate drug targets in the treatment, diagnosis, and prognosis of colorectal cancer. According to the results of our research, it can be said that mTOR1 and UCA1 probably play an important role in the development of colorectal cancer. Increased expression of UCA1 in tumor samples may show a significant association with mTOR1, since mTOR1 expression has also increased. Similar to Wu and Luo (24) and Wang and Fa's study (25), which showed the relationship between mTOR1 and UCA1 in different cancers, we also concluded that there is a relationship between mTOR1/UCA1 and colorectal cancer in Iranian patients.

\section{Footnotes}

Authors' Contribution: M. A. collected the clinical data, interpreted them and drafted the manuscript. M. S. conceived and designed the evaluation and prepared the manuscript.

Conflict of Interests: The authors declare there is no conflict of interest in this article.

Data Reproducibility: The data presented in this study are openly available in one of the repositories or will be available on request from the corresponding author by this journal representative at any time during submission 
or after publication. Otherwise, all consequences of possible withdrawal or future retraction will be with the corresponding author.

Ethical Approval: Research protocol was approved by the Ethics Committee of Islamic Azad Tehran Medical Sciences University-Pharmacy and Pharmaceutical Branches Faculty (ID number: IR.IAU.PS.REC.1399.085).

Funding/Support: Thanks to Tehran Medical Sciences ISLAMIC AZAD UNIVERSITY, which supported this research.

Informed Consent: Written informed consents were obtained from patients of all subjects.

\section{References}

1. Wild CP, Stewart BW, Wild C. World cancer report 2014. Lyon, France: International Agency for Research on Cancer; 2014.

2. Brody H. Colorectal cancer. Nature. 2015;521(7551). S1. doi: 10.1038/521S1a. [PubMed: 25970450].

3. Siegel RL, Miller KD, Fedewa SA, Ahnen DJ, Meester RGS, Barzi A, et al. Colorectal cancer facts and figures 2017-2019. Atlanta, USA: American Cancer Society; 2017.

4. Levin B, Lieberman DA, McFarland B, Andrews KS, Brooks D, Bond J, et al. Screening and surveillance for the early detection of colorectal cancer and adenomatous polyps, 2008: a joint guideline from the American Cancer Society, the US Multi-Society Task Force on Colorectal Cancer, and the American College of Radiology. Gastroenterology. 2008;134(5):1570-95. doi: 10.1053/j.gastro.2008.02.002. [PubMed: 18384785].

5. Murphy G, Devesa SS, Cross AJ, Inskip PD, McGlynn KA, Cook MB. Sex disparities in colorectal cancer incidence by anatomic subsite, race and age. Int J Cancer. 2011;128(7):1668-75. doi: 10.1002/ijc.25481. [PubMed: 20503269]. [PubMed Central: PMC3031675].

6. Doubeni CA, Laiyemo AO, Major JM, Schootman M, Lian M, Park Y, et al. Socioeconomic status and the risk of colorectal cancer: An analysis of more than a half million adults in the National Institutes of Health-AARP Diet and Health Study. Cancer. 2012;118(14):363644. doi: 10.1002/cncr.26677. [PubMed: 22898918]. [PubMed Central: PMC3422782].

7. Doubeni CA, Major JM, Laiyemo AO, Schootman M, Zauber AG, Hollenbeck AR, et al. Contribution of behavioral risk factors and obesity to socioeconomic differences in colorectal cancer incidence. J Natl Cancer Inst. 2012;104(18):1353-62. doi: 10.1093/jnci/djs346. [PubMed: 22952311]. [PubMed Central: PMC3529596].

8. Zumkeller N, Brenner H, Zwahlen M, Rothenbacher D. Helicobacter pylori infection and colorectal cancer risk: A meta-analysis. Helicobacter. 2006;11(2):75-80. doi: 10.1111/j.1523-5378.2006.00381.x. [PubMed: 16579836].

9. Robertson DJ. $\mathrm{ABC}$ of Colorectal Cancer. Gastroenterology. 2012;143(3):868-9. doi:10.1053/j.gastro.2012.07.090.

10. Poschl G, Seitz HK. Alcohol and cancer. Alcohol Alcohol. 2004;39(3):15565. doi: 10.1093/alcalc/agh057. [PubMed: 15082451].

11. Johns LE, Houlston RS. A systematic review and meta-analysis of familial colorectal cancer risk. Am J Gastroenterol. 2001;96(10):2992-3003. doi:10.1111/j.1572-0241.2001.04677.x. [PubMed: 11693338].

12. Goddard AF, James MW, McIntyre AS, Scott BB; British Society of Gastroenterology. Guidelines for the management of iron deficiency anaemia. Gut. 2011;60(10):1309-16. doi:10.1136/gut.2010.228874. [PubMed: 21561874].

13. Rockey DC, Cello JP. Evaluation of the gastrointestinal tract in patients with iron-deficiency anemia. N Engl J Med. 1993;329(23):1691-5. doi:10.1056/NEJM199312023292303. [PubMed: 8179652]
14. Deverakonda A. Diagnosis and treatment of colorectal cancer: A review. Res Rev J Med Health Sci. 2016;5:1-15.

15. Fearon ER, Vogelstein B. A genetic model for colorectal tumorigenesis. Cell. 1990;61(5):759-67. doi: 10.1016/0092-8674(90)90186-i. [PubMed: 2188735].

16. Han Y, Yang YN, Yuan HH, Zhang TT, Sui H, Wei XL, et al. UCA1, a long non-coding RNA up-regulated in colorectal cancer influences cell proliferation, apoptosis and cell cycle distribution. Pathology. 2014;46(5):396-401. doi: 10.1097/PAT.0000000000000125. [PubMed: 24977734].

17. Xue M, Chen W, Xiang A, Wang R, Chen H, Pan J, et al. Hypoxic exosomes facilitate bladder tumor growth and development through transferring long non-coding RNA-UCA1. Mol Cancer. 2017;16(1):143. doi: 10.1186/s12943-017-0714-8. [PubMed: 28841829]. [PubMed Central: PMC5574139].

18. Xue M, Li X, Li Z, Chen W. Urothelial carcinoma associated 1 is a hypoxia-inducible factor-1alpha-targeted long noncoding RNA that enhances hypoxic bladder cancer cell proliferation, migration, and invasion. Tumour Biol. 2014;35(7):6901-12. doi: 10.1007/s13277-014-1925x. [PubMed: 24737584].

19. Ni B, Yu X, Guo X, Fan X, Yang Z, Wu P, et al. Increased urothelial cancer associated 1 is associated with tumor proliferation and metastasis and predicts poor prognosis in colorectal cancer. Int J Oncol. 2015;47(4):1329-38. doi: 10.3892/ijo.2015.3109. [PubMed: 26238511].

20. Su EC, Chen YS, Tien YC, Liu J, Ho BC, Yu SL, et al. ChemiRs: a web application for microRNAs and chemicals. BMC Bioinformatics. 2016;17:167. doi: 10.1186/s12859-016-1002-0. [PubMed: 27091357]. [PubMed Central: PMC4836156].

21. Forbes SA, Bindal N, Bamford S, Cole C, Kok CY, Beare D, et al. COSMIC: mining complete cancer genomes in the Catalogue of Somatic Mutations in Cancer. Nucleic Acids Res. 2011;39(Database issue):D94550. doi: 10.1093/nar/gkq929. [PubMed: 20952405]. [PubMed Central: PMC3013785].

22. Kennedy BK, Lamming DW. The Mechanistic Target of Rapamycin: The Grand ConductOR of Metabolism and Aging. Cell Metab. 2016;23(6):990-1003. doi: 10.1016/j.cmet.2016.05.009. [PubMed: 27304501]. [PubMed Central: PMC4910876].

23. Jiang BH, Liu LZ. Role of mTOR in anticancer drug resistance: Perspectives for improved drug treatment. Drug Resist Updat. 2008;11(3):6376. doi: 10.1016/j.drup.2008.03.001. [PubMed: 18440854]. [PubMed Central: PMC2519122].

24. Wu C, Luo J. Long Non-Coding RNA (lncRNA) Urothelial CarcinomaAssociated 1 (UCA1) Enhances Tamoxifen Resistance in Breast Cancer Cells via Inhibiting mTOR Signaling Pathway. Med Sci Monit. 2016;22:3860-7. doi: 10.12659/msm.900689. [PubMed: 27765938]. [PubMed Central: PMC5077288].

25. Wang X, Fa X. Knockdown of UCA1 inhibits viability and glycolysis by suppressing PKM2 expression through the mTOR pathway in nonsmall cell lung cancer cells. RSC Advances. 2018;8(19):10610-9. doi: 10.1039/c8ra00860d.

26. Huarte $M$. The emerging role of IncRNAs in cancer. Nat Med. 2015;21(11):1253-61. doi: 10.1038/nm.3981. [PubMed: 26540387].

27. Yang X, Liu W, Xu X, Zhu J, Wu Y, Zhao K, et al. Downregulation of long noncoding RNA UCA1 enhances the radiosensitivity and inhibits migration via suppression of epithelialmesenchymal transition in colorectal cancer cells. Oncol Rep. 2018;40(3):1554-64. doi: 10.3892/or.2018.6573. [PubMed:30015983].

28. Cui M, Chen M, Shen Z, Wang R, Fang X, Song B. LncRNA-UCA1 modulates progression of colon cancer through regulating the miR-285p/HOXB3 axis. J Cell Biochem. 2019. doi: 10.1002/jcb.27630. [PubMed: 30652355].

29. Populo H, Lopes JM, Soares P. The mTOR signalling pathway in human cancer. Int J Mol Sci. 2012;13(2):1886-918. doi: 10.3390/ijms13021886. [PubMed: 22408430]. [PubMed Central: PMC3291999]. 
30. Hanahan D, Weinberg RA. Hallmarks of cancer: The next generation. Cell. 2011;144(5):646-74. doi: 10.1016/j.cell.2011.02.013. [PubMed: 21376230].

31. Melling N, Simon R, Izbicki JR, Terracciano LM, Bokemeyer C, Sauter $\mathrm{G}$, et al. Expression of phospho-mTOR kinase is abundant in colorectal cancer and associated with left-sided tumor localization. Int J Clin Exp Pathol. 2015;8(6):7009-15. [PubMed: 26261591]. [PubMed Central: PMC4525925].

32. Zhang YJ, Dai Q, Sun DF, Xiong H, Tian XQ, Gao FH, et al. mTOR signal- ing pathway is a target for the treatment of colorectal cancer. Ann Surg Oncol. 2009;16(9):2617-28. doi: 10.1245/s10434-009-0555-9. [PubMed: 19517193].

33. Gulhati P, Bowen KA, Liu J, Stevens PD, Rychahou PG, Chen M, et al. mTORC1 and mTORC2 regulate EMT, motility, and metastasis of colorectal cancer via RhoA and Rac1 signaling pathways. Cancer Res. 2011;71(9):3246-56. doi: 10.1158/0008-5472.CAN-10-4058. [PubMed: 21430067]. [PubMed Central: PMC3085654]. 